\title{
Altered Exression of Circadian Clock genes in Polyglandular Autoimmune Syndrome type III
}

* equally distributed

Anna Angelousi ${ }^{1 *}$, Narjes Nasiri-Ansari ${ }^{2}{ }^{*}$, Eliana Spilioti $^{2}$, Vasiliki Kalotychou $^{3}$, George Chrousos $^{4}$, Gregory Kaltsas $^{1}$, Eva Kassi $^{2}$

${ }^{1}$ Department of Pathophysiology, Unit of Endocrinology, National and Kapodistrian University of Athens, ${ }^{2}$ Department of Biological Chemistry,

National and Kapodistrian University of Athens Hospital, ${ }^{3}$ 1st Department of Internal Medicine, University of Athens, School of Medicine, Laiko hospital, ${ }^{4}$ First Department of Pediatrics, School of Medicine, University of Athens, Greece.

\section{Introduction}

CLOCK system is a highly conserved, ubiquitous molecular "clock" which creates internal circadian rhythmicity under the influence of light/dark information. CLOCK system is regulated by the coordinated activation/inactivation of several transcription factors, including the CLOCK, the BMAL1 and other essential regulators, such as the Pers, Crys and RORs. The present study aimed to evaluate the circadian rhythm of clock-related genes expressed in patients with polyglandular autoimmune syndrome type III (PASIII).

\section{Methods}

Nineteen patients diagnosed with PASIII ( 5 males) and 12 healthy controls (4 males) were enrolled. The characteristics of the participants are shown in Table 1 . All patients had normal response to Synacthen test. By performing real-time PCR, we analysed mRNA expression of CLOCK-related genes (CLOCK, $B M A L 1, R O R, P e r 3$ and GILZ) and glucocorticoid receptor (GR) gene in peripheral blood mononuclear cells (PBMCs) isolated by Lymphoprep density gradient centrifugation from blood samples drawn at 8 am and $8 \mathrm{pm}$. GR protein expression was analysed by Western Blot.

At the same time, serum cortisol and plasma ACTH were measured by chemiluminescence.

\section{Results}

$\diamond \quad$ No statistical differences were found in cortisol, ACTH and TSH plasma levels between patients and controls.

\begin{tabular}{|c|c|c|c|c|c|c|c|c|c|}
\hline |Characteristics & \multicolumn{3}{|c|}{ Patients } & \multicolumn{4}{|c|}{ Controls } & & \multirow{2}{*}{$\frac{\mathbf{p}^{*}}{\mathrm{~ns}}$} \\
\hline Total (N) & \multicolumn{3}{|c|}{19} & & \multicolumn{2}{|c|}{12} & & & \\
\hline Age(median,yrs) & \multicolumn{2}{|c|}{55} & & & \multicolumn{2}{|c|}{52} & & & ns \\
\hline \multirow{2}{*}{$\operatorname{Sex}(m / f)$} & \multicolumn{2}{|c|}{$5 / 14$} & & & \multicolumn{2}{|c|}{$4 / 8$} & & & $\mathrm{~ns}$ \\
\hline & am & pm & $\begin{array}{l}\Delta \mathrm{CT} \\
\mathrm{pm} / \mathrm{am}\end{array}$ & $\begin{array}{l}\text { p(pm } \\
\text { vs.am) }\end{array}$ & am & pm & $\begin{array}{l}\Delta \mathrm{CT} \\
\mathrm{pm} / \mathrm{am}\end{array}$ & $\begin{array}{l}\text { p(pm } \\
\text { vs.am) }\end{array}$ & \\
\hline Mean F( $\mu \mathrm{g} / \mathrm{dl})$ & $19.4 \pm 4.4$ & $5.2 \pm 3.4$ & & 0.001 & $16.5 \pm 3.5$ & $5 \pm 2.9$ & & 0.01 & ns \\
\hline Mean ACTH $(\mathrm{pg} / \mathrm{ml})$ & $14.7 \pm 5.3$ & $10 \pm 7.5$ & & 0.002 & $17 \pm 9$ & $11 \pm 9$ & & 0.1 & ns \\
\hline $\begin{array}{l}\text { Mean TSH }(\mathrm{U} / \mathrm{ml}) \\
\text { Genes (median } \\
\Delta \mathrm{CT})\end{array}$ & $2.7 \pm 1.4$ & - & & & $1.8 \pm 1.2$ & - & & & $\mathrm{ns}$ \\
\hline$-\mathrm{GR}^{\prime}$ & -0.35 & -0.6 & 1.04 & 0.1 & -0.04 & 0.83 & 0.80 & 0.67 & 0.05 \\
\hline- CLOCK & 5.6 & 4.0 & 0.74 & 0.03 & 3.91 & 4.40 & 0.96 & 0.52 & 0.018 \\
\hline -BMAL1 & 4.9 & 3.6 & 0.52 & 0.04 & 4.08 & 4.38 & 1.06 & 0.14 & 0.033 \\
\hline- ROR & 0.6 & 0.35 & 0.27 & 0.8 & 0.16 & 1.26 & 0.478 & 0.12 & 0.1 \\
\hline -PER3 & 6.07 & 7.22 & 0.98 & 0.9 & 6.63 & 7.27 & 1.1 & 0.03 & 0.05 \\
\hline & & & & & & & & & \\
\hline
\end{tabular}

Table 1. Characteristics of the patients and controls. Differences in the biochemical markers and

the mRNA expression $(\triangle C T)$ of the 6 CLOCK related genes between mesurement in the morning

and the evening as well as differences between patients with PASS III and controls.

* patients vs. controls

$\diamond \quad$ An overexpression of the evening CLOCK and BMAL1 genes compared to the morning- was observed in patients (Fig.1,2) .
Figure 1

CLOCK in controls

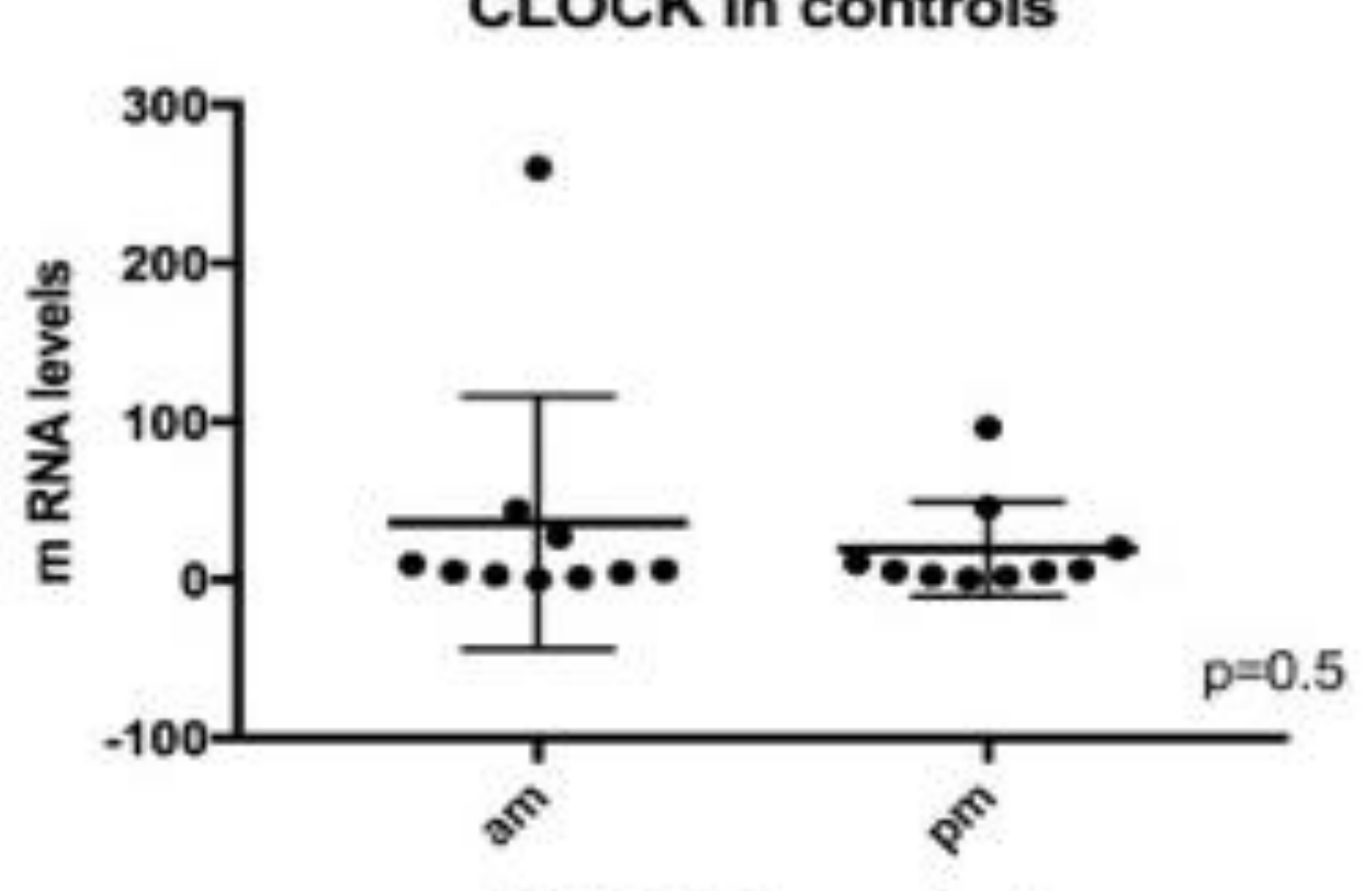

BMAL1 in controls

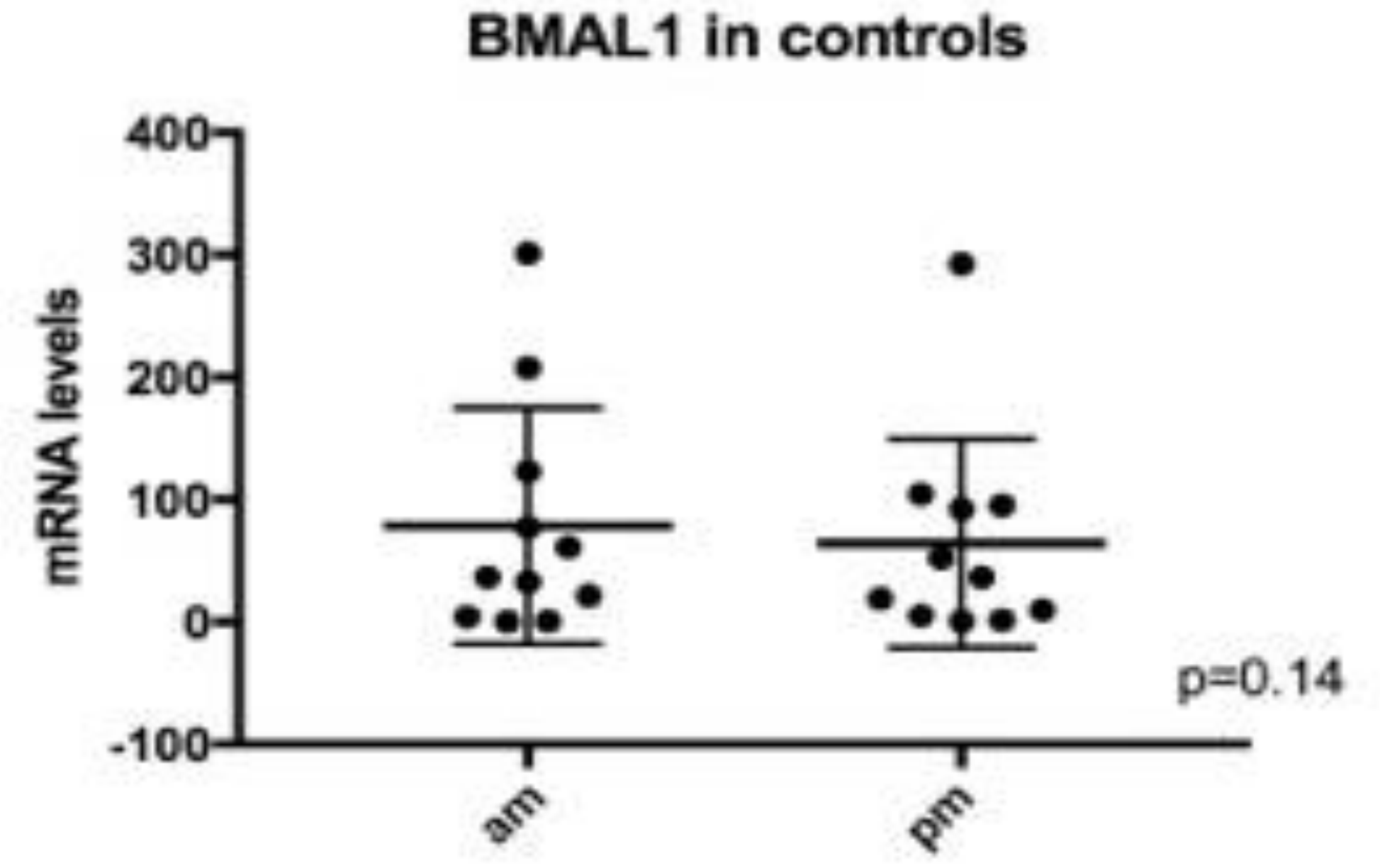

Figure 2

CLOCK in patients
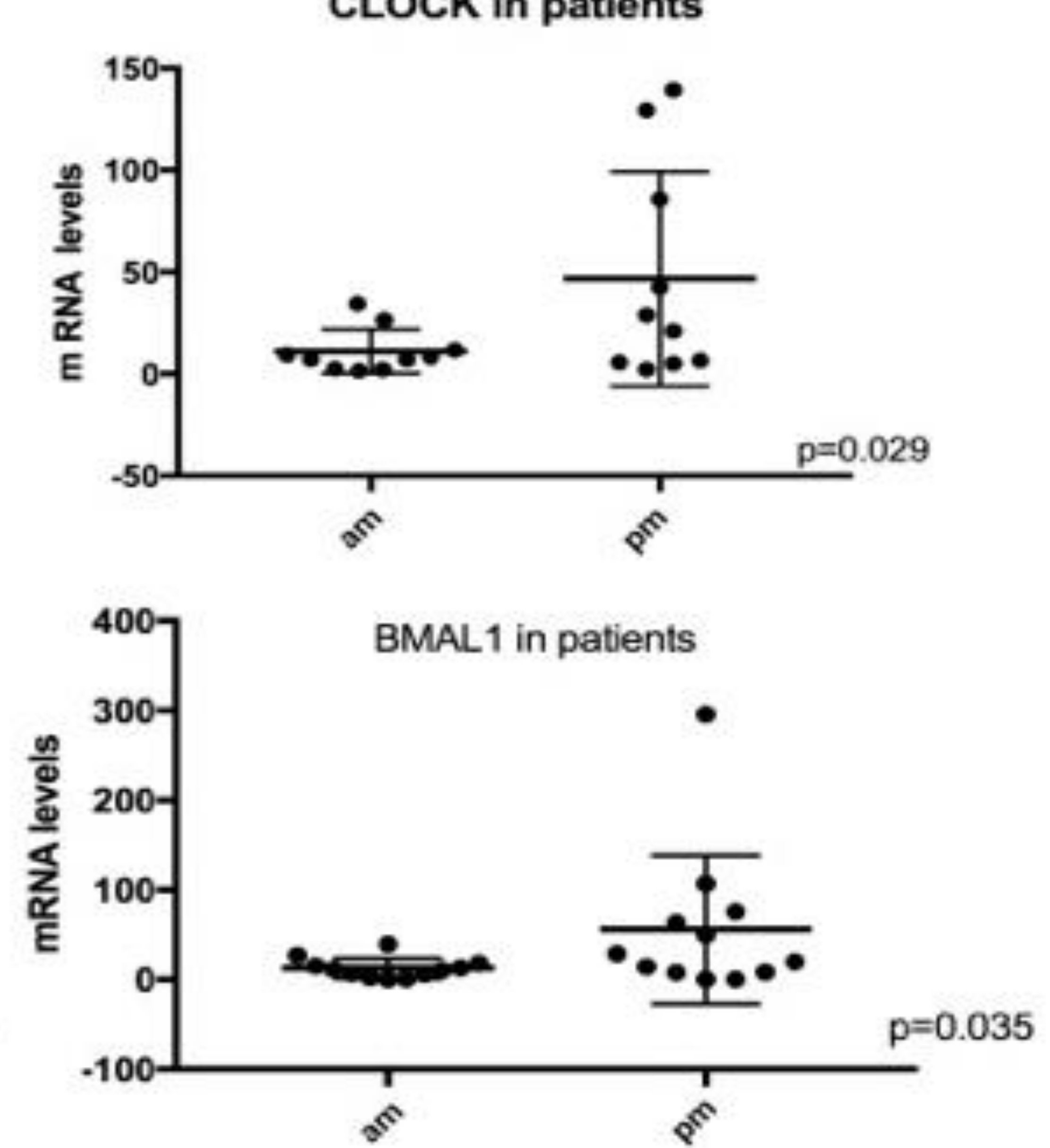

$\diamond \quad$ Controls exhibited a significant overexpression of the PER3 gene in the morning compared to the evening.

$\diamond \quad$ Patients exhibited a significantly lower mRNA ratio $\left(R_{p m / a m}\right)$ of GR, CLOCK, BMAL1, and PER3 compared to controls (Fig. 3,4, 5, 6).
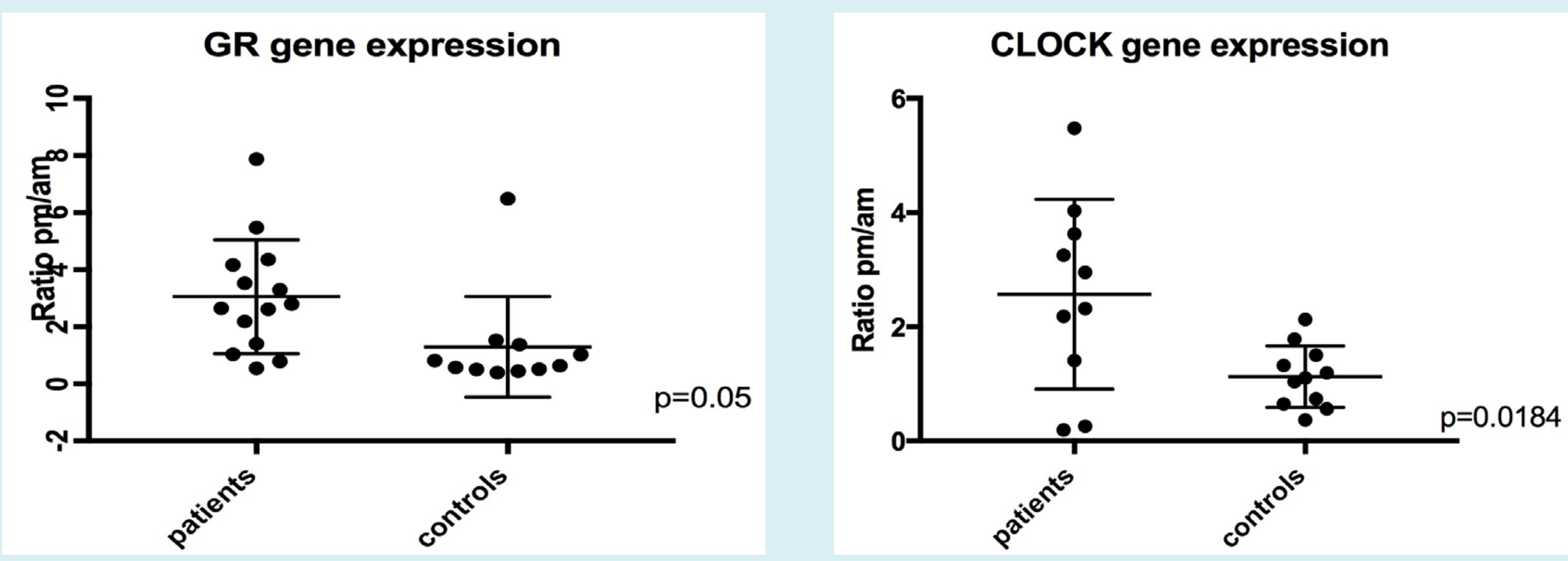

Figure 3

Figure 4

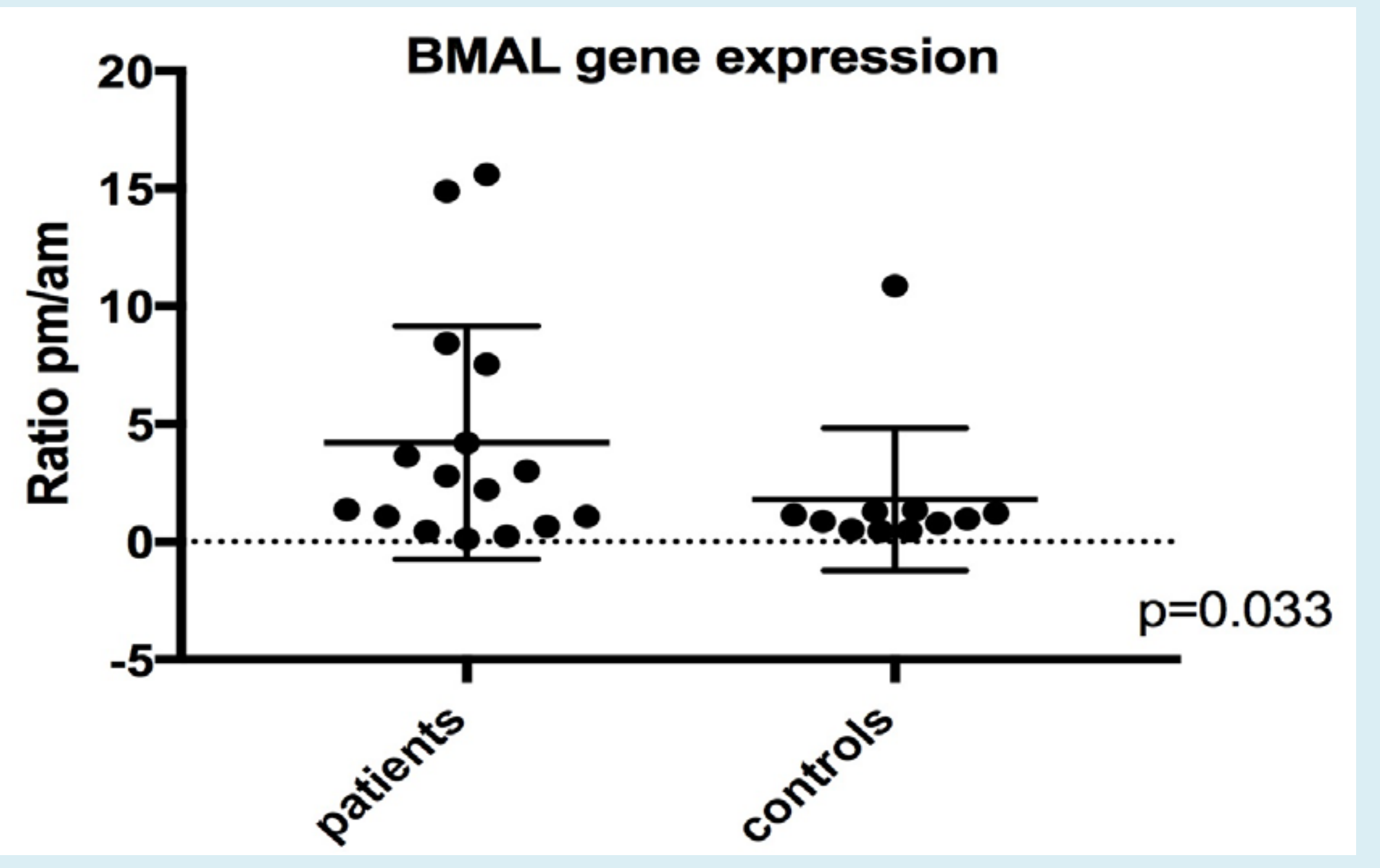

Figure 5

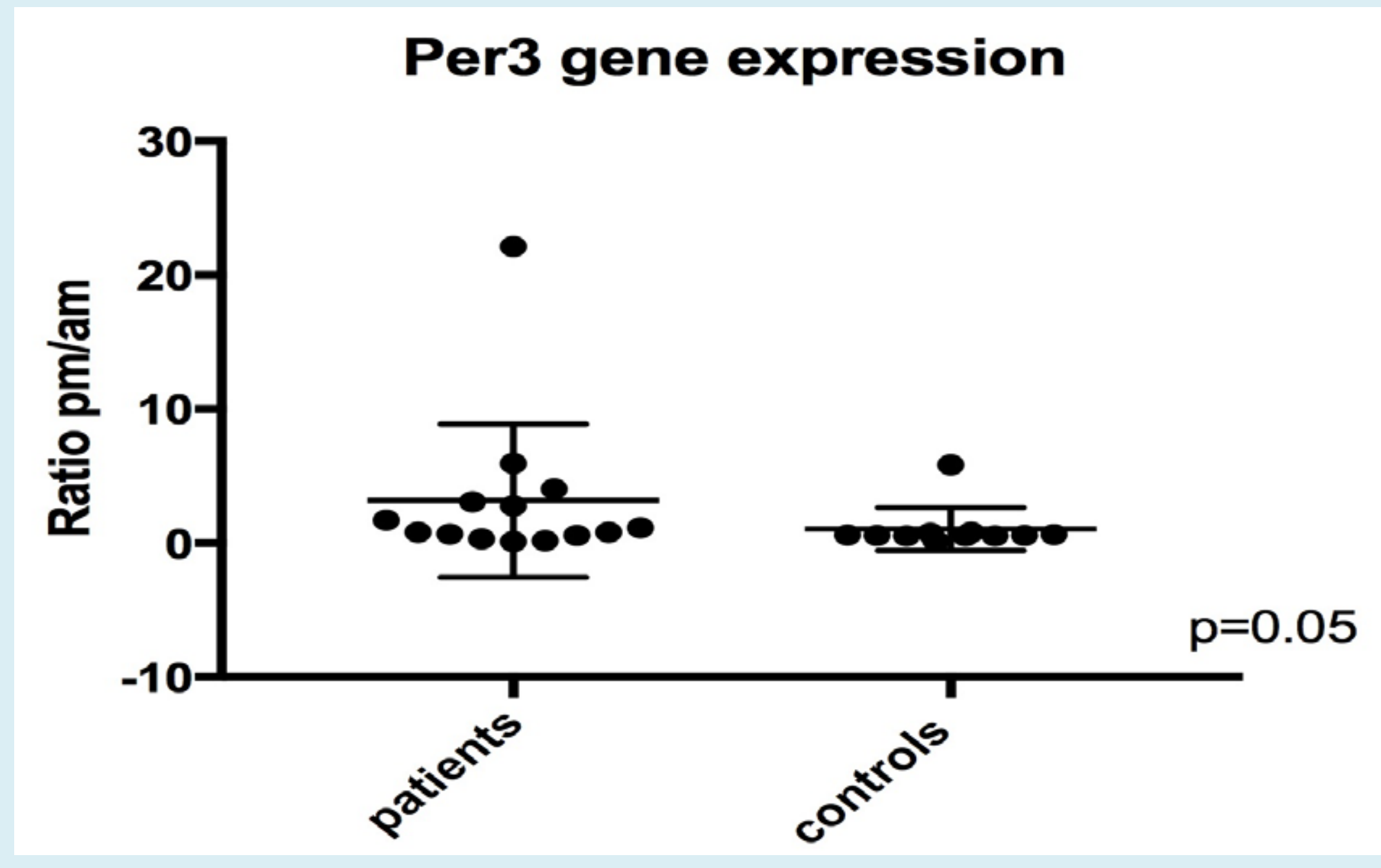

Figure 6 $\diamond \quad$ Cortisol circadian variation $\left(\Delta \mathrm{F}_{\mathrm{pm} / \mathrm{am}}\right)$ demonstrated a significant positive correlation with the mRNA ratio $\left(\mathrm{R}_{\mathrm{pm} / \mathrm{am})}\right.$ of GILZ. Table 2

\begin{tabular}{|c|c|c|c|c|c|c|}
\hline & $\begin{array}{l}\Delta \mathrm{CT} \mathrm{pm} / \mathrm{am} \\
\text { GR }\end{array}$ & $\begin{array}{l}\Delta \mathrm{ACT} \mathrm{pm} / \mathrm{am} \\
\text { CLOCK }\end{array}$ & $\begin{array}{l}\Delta \mathrm{ACT} \mathrm{pm} / \mathrm{am} \\
\text { BMAL1 }\end{array}$ & $\begin{array}{l}\Delta \mathrm{CT} \mathrm{pm} / \mathrm{am} \\
\text { Per3 }\end{array}$ & $\begin{array}{l}\Delta \mathrm{CT} \mathrm{pm} / \mathrm{am} \\
\text { ROR }\end{array}$ & $\begin{array}{l}\Delta \mathrm{CT} \mathrm{pm} / \mathrm{am} \\
\text { GILZ }\end{array}$ \\
\hline $\begin{array}{l}\text { Patients } \\
\text { F pm/am }\end{array}$ & $\begin{array}{l}\mathrm{F}=0.12(-0.4-0.6) \\
\mathrm{p}=0.6\end{array}$ & $\begin{array}{l}\mathrm{f}=0.34(-0.22-0.7) \\
p=0.2\end{array}$ & $\begin{array}{l}\mathrm{r}=0.29(-0.2-0.6) \\
\mathrm{p}=0.2\end{array}$ & $\begin{array}{l}\mathrm{g}=0.21(-0.29-0.6) \\
\mathrm{D}=0.4\end{array}$ & $\begin{array}{l}\mathrm{F}=0.46, \mathrm{p}=0.06 \\
\mathrm{p}=0.06\end{array}$ & $\begin{array}{l}\mathrm{F}=0.7(0.34-0.9) \\
\mathrm{D}=0.002\end{array}$ \\
\hline $\begin{array}{l}\text { Controls } \\
\text { F pm/am }\end{array}$ & $\begin{array}{l}\mathrm{F}=0.7(-0.9-0.19) \\
\mathrm{D}=0.53\end{array}$ & $\begin{array}{l}x=0.18(-0.45-0.69) \\
p=0.5\end{array}$ & $\begin{array}{l}0.2(-0.4-0.75) \\
p=0.43\end{array}$ & $\begin{array}{l}E=0.41(0.22-0.8) \\
B=0.18\end{array}$ & $\begin{array}{l}r=0.03(-0.57-0.6) \\
D=0.95\end{array}$ & $\begin{array}{l}E=0.4(-0.79-0.23) \\
D=0.2\end{array}$ \\
\hline
\end{tabular}

Table 2. Correlations among mRNA levels (ratio of $\triangle C T \mathrm{~mm} / \mathrm{am}$ ) of GR CLOCK BMALL PER3.ROR. GILZ with the ratio pm/am of cortisol(E).

\section{Western Blot analysis}
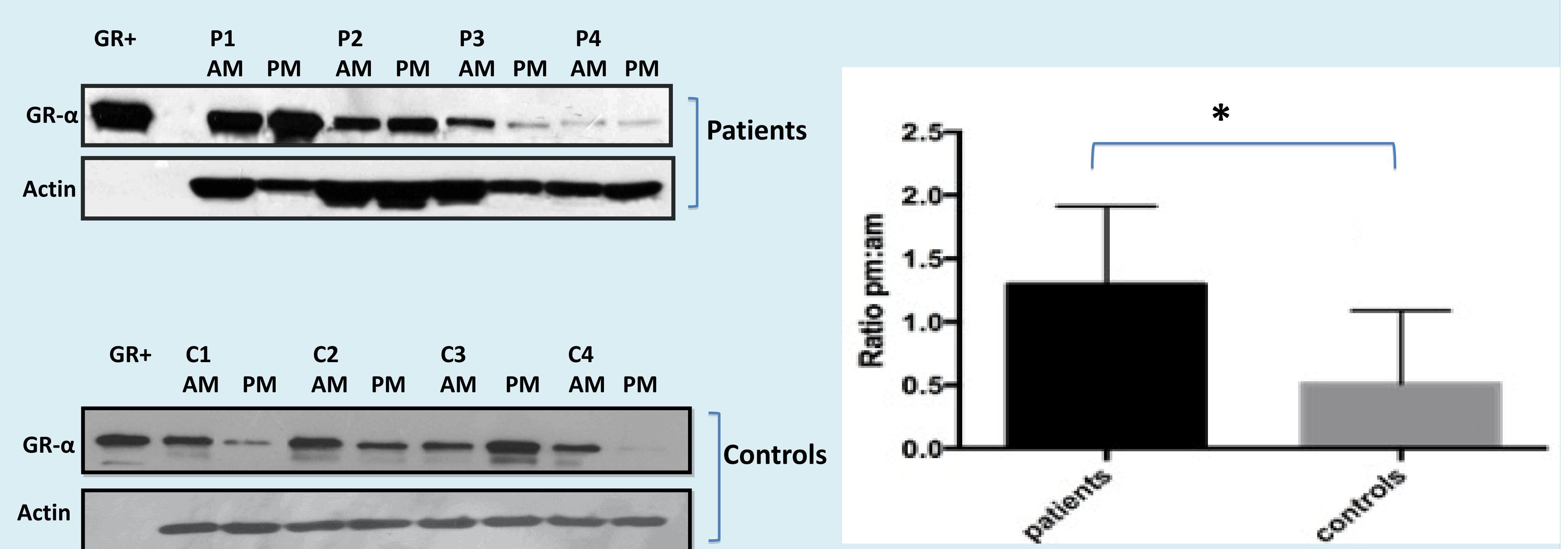

Western blot analysis revealed a significant greater slope of the GR- $\alpha$ protein level in the evening in control group compared to patient group.

\section{Conclusions}

These findings suggest that there is an abberant expression of clock-related genes in patients with PASIII compared to healthy controls. Daily pattern expression of the 6 circadian clock genes was disrupted in patients with PAS III indicating a possible association with the pathogenesis of the disease. 